\title{
The population point of view on the evolution of TeV pulsar wind nebulae
}

\author{
S. Klepser ${ }^{* a}$, Y. Gallant ${ }^{b}$, M. Mayer ${ }^{c}$, K. Valerius ${ }^{d}$ for the H.E.S.S. collaboration \\ ${ }^{a}$ DESY, D-15738 Zeuthen, Germany \\ ${ }^{b}$ Laboratoire Univers et Particules de Montpellier, Université Montpellier, CNRS/IN2P3, CC 72, \\ Place Eugène Bataillon, F-34095 Montpellier Cedex 5, France \\ ${ }^{c}$ Institut für Physik, Humboldt-Universität zu Berlin, Newtonstr. 15, D 12489 Berlin, Germany \\ ${ }^{d}$ Karlsruhe Institute of Technology, Institute for Nuclear Physics, P.O. Box 3640, D-76021 \\ Karlsruhe, Germany
}

\begin{abstract}
To investigate the nature and evolution of $\mathrm{TeV}$ pulsar wind nebulae, we examine the firmly identified PWNe in the H.E.S.S. Galactic Plane Survey, along with the few other known detections from the literature, as well as the upper limits extracted from the H.E.S.S survey. These data exhibit a correlation of $\mathrm{TeV}$ surface brightness with pulsar spin-down power. It appears to be caused by both an increase of $\mathrm{TeV}$ extension and a decrease of $\mathrm{TeV}$ luminosity with decreasing spin-down power. We also find that the offsets of pulsars with ages around $10 \mathrm{kyr}$ with respect to the wind nebula centres are frequently larger than can be plausibly explained by pulsar proper motion and could be due to an asymmetric environment. These and other results will be presented and put to context with a basic modelling of $\mathrm{TeV}$ pulsar wind nebula evolution.
\end{abstract}

35th International Cosmic Ray Conference - ICRC2017

10-20 July, 2017

Bexco, Busan, Korea

${ }^{*}$ Speaker. 
This conference contribution is based on the recent paper on the subject (H.E.S.S. Collaboration et al. 2017 [1]). All details, more plots, and references can be found in that reference. This proceedings paper wraps up the main conclusions and key figures of the paper.

\section{Overview}

The paper subsumes and examines the population of $\mathrm{TeV}$ pulsar wind nebulae (PWNe) found to date. An updated census presents 14 objects reanalysed in the H.E.S.S. Galactic Plane Survey (HGPS) pipeline, which are considered to be firmly identified PWNe. Five more objects could be found outside that catalogue range or pipeline. In an evaluation of candidate $\mathrm{PWNe}$, we conclude that there are ten strong further candidates in the HGPS data.

Most of the PWNe are located in the bright and dense Crux Scutum arm of the inner Milky Way (Fig. 1). A spatial correlation study confirmed the picture drawn in earlier studies, namely that only young, energetic pulsars grow $\mathrm{TeV}$ pulsar wind nebulae that are bright enough for detection with presently available Cherenkov telescopes. For the first time, flux upper limits for undetected PWNe are given around 22 pulsars with a spin-down power beyond $10^{35} \mathrm{erg} \mathrm{s}^{-1}$ and with expected apparent extensions (plus offsets) below $0.6^{\circ}$ in the sky.

\section{Census of PWNe around high- $\dot{E}$ pulsars}

Of the 17 most energetic ATNF pulsars, with a spin-down power of $\dot{E} \geq 10^{37} \mathrm{erg} \mathrm{s}^{-1}, 11$ have either an identified $\mathrm{TeV}$ wind nebula (9) or candidate (2) featured in the present study. Of the remaining 6 ,

- 3 are included in Table 5 in H.E.S.S. Collaboration et al. 2017 [1], where all flux limits of pulsars without detected PWN are listed;

- 3 are out of the range of the HGPS:

- PSR J2022+3842: SNR G076.9+01.0, contains an X-ray PWN; not reported in TeV

- PSR J2229+6114: Boomerang, contains an X-ray PWN; detected by MILAGRO and VERITAS, but of unclear nature in $\mathrm{TeV}$

- J0540-6919: In the Large Magellanic Cloud; a limit is given in H.E.S.S. Collaboration et al. 2015 [2].

Concluding, only 5 of the 17 highest- $\dot{E}$ pulsars remain without a detected potential counterpart in the TeV band.

\section{PWN evolution}

Figures 5 to 10 in H.E.S.S. Collaboration et al. 2017 [1] show, like Fig. 2 and Fig. 3 in this proceedings paper, a variety of trends between pulsar and $\mathrm{TeV}$ wind nebula parameters, and consistently compare them to a simple one-zone time-dependent emission model of the $\mathrm{TeV}$ emission with a varied range of model input parameters. The main conclusion is that for several observables, 

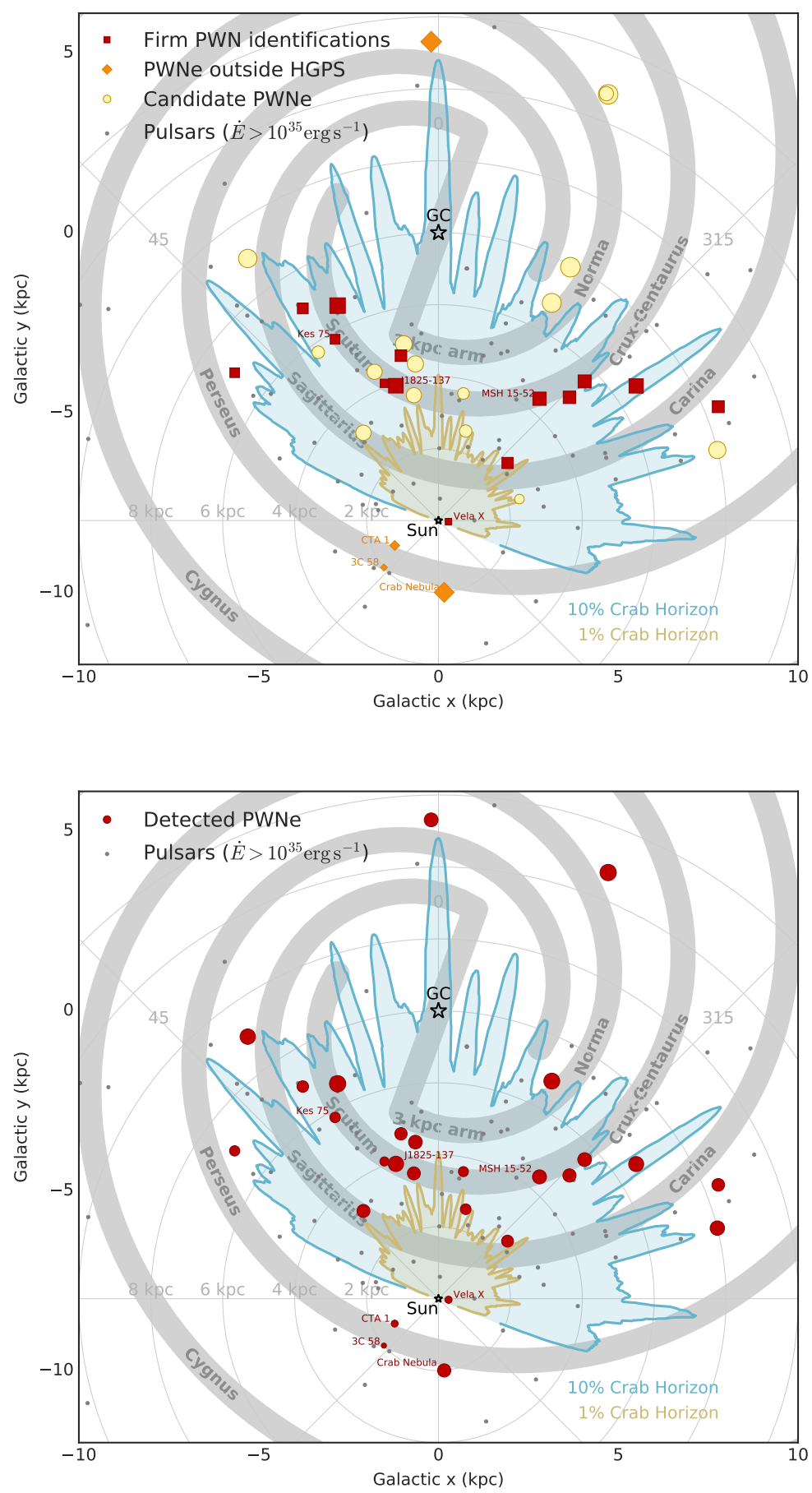

Figure 1: Schematic of the objects discussed here in the context of the Milky Way and its spiral arms. The yellow and blue curves outline the sensitivity horizon of the HGPS for point-like sources with an integrated gamma-ray luminosity (1-10 TeV) of $1 \%$ and $10 \%$ of the Crab luminosity, respectively. Top: Firmly identified PWNe, candidates, and energetic pulsars $\left(\dot{E}>10^{35} \mathrm{erg} \mathrm{s}^{-1}\right)$ without detected TeV wind nebula. Bottom: Simplified view with all firm (HGPS and external) identifications and positively rated candidates displayed with the same symbol, and negative candidates removed. The figures are reproduced from H.E.S.S. Collaboration et al. $2017^{1}$, but the sizes of the symbols are scaled with $\log L_{1-10 \mathrm{TeV}}$ here. 
a trend was found in the data that is consistent with the trends suggested by our model. With a moderate variation of the model input parameters, we can mimic also the spreads of the observables. Our first-order understanding of the evolution of $\mathrm{TeV}$ pulsar wind nebulae with ages up to some tens of kiloyears therefore seems to be compatible with what the whole population of detected and undetected PWNe suggests.

More concretely, using the flux limits for undetected PWNe, we find evidence that the $\mathrm{TeV}$ luminosity of PWNe decays with time while they expand in size, preventing the detection of those whose pulsar has dropped below a spin-down of $\sim 10^{36} \mathrm{erg} \mathrm{s}^{-1}$ (roughly corresponding to several tens of kiloyears). This was implicitly known before from the mere non-detection of old $\mathrm{TeV}$ pulsar wind nebulae, but for the first time could be put into a quantitative perspective, both by fitting data and limits, and by comparing the data to model predictions. The power-law relation between $\mathrm{TeV}$ luminosity and pulsar spin-down could be estimated as $L_{1-10 \mathrm{TeV}} \sim \dot{E}^{0.58 \pm 0.21}$, in consistency with the model, which suggests a power index of around 0.5 .
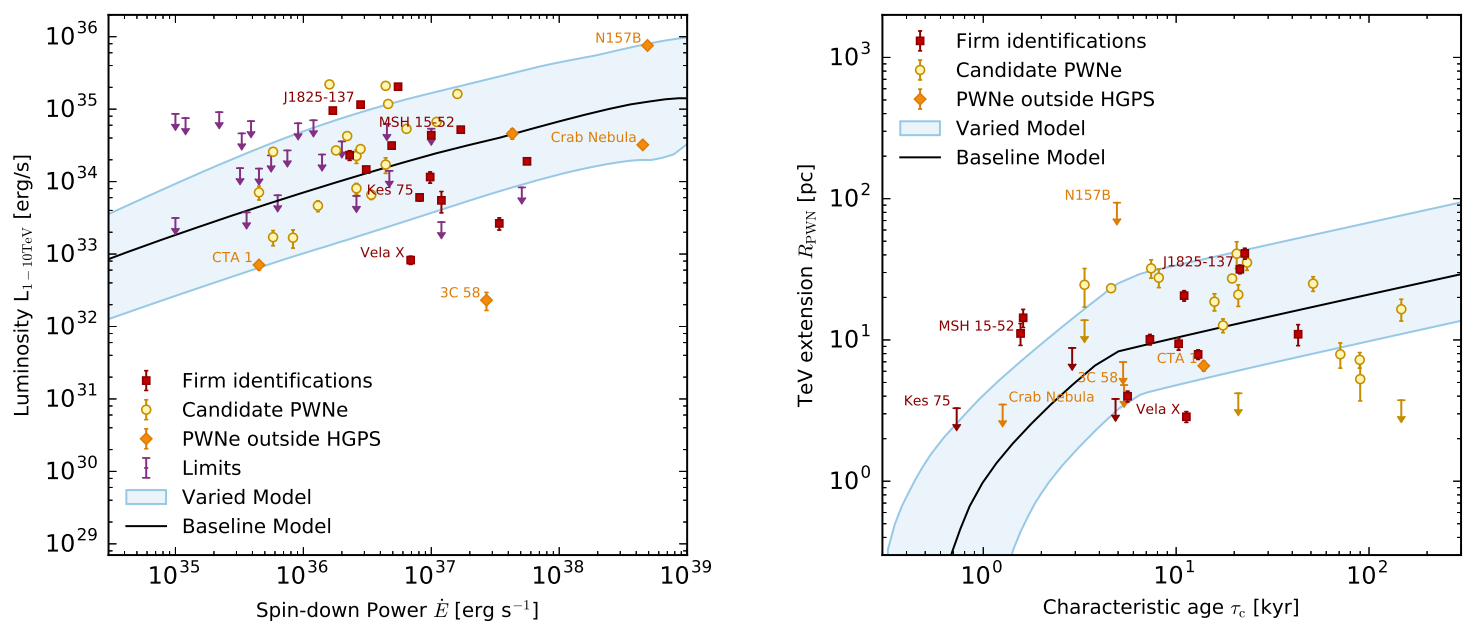

Figure 2: Left: Relation of TeV luminosity and pulsar $\dot{E}$. Right: PWN extension evolution with time, in comparison to the modelling considered in this work. The figures are reproduced from H.E.S.S. Collaboration et al. 2017 [1].

Another feature that was discussed on some individual objects before (e.g. [3]) is the "crushing" of PWNe, which can be exerted by the inward-bound reverse front of the supernova shock wave. For SNRs that develop asymmetrically, for instance due to an inhomogeneous surrounding medium, this crushing may result in distortion and displacement of the wind nebula. A very bright, very extended example of this is HESS J1825-137, also presented at this conference [4]. Put to a population-scoped context, it becomes clear that pulsar proper motions alone are not sufficient to explain the large offsets observed, and some kind of a crushing mechanism may indeed be the dominant and frequent cause of pulsar-PWN offset in middle-aged systems. Furthermore, the offsets of PWNe from their pulsars appear to relate to high efficiency (Fig. 3, right), suggesting that the PWNe either gain energy and brightness through the process that causes the offset or that dense surroundings amplify both the IC luminosity and the offset between pulsar and wind nebula. While the evidence for this at present is not very strong, following up with expanded future studies is certainly worthwhile. 
The expansion of PWNe with time was also shown to be evident in the data. The fitted relation $R \sim \tau_{\mathrm{c}}^{0.55 \pm 0.23}$ suggests an average expansion coefficient in between those expected in theory $(1.2$ and 0.3). The data set is not comprehensive enough to do a fit with two power laws, but appears to be consistent with the model (Fig. 2, right). Notably, this expansion is not so clear in X-rays, where the synchrotron emission always remains very local because it only traces the young particles in areas of high magnetic field relatively close to the pulsar. Most of the old objects ( $>30 \mathrm{kyr})$ are therefore smaller than $1 \mathrm{pc}$ in their bright $\mathrm{X}$-ray core emission.

As a consequence of the two moderate correlations of luminosity and spatial extent with pulsar $\dot{E}$, a stronger correlation was found between the PWN surface brightness and pulsar $\dot{E}$ (Fig. 3, left). What stands out is not only the correlation itself, but also its relatively low scatter.
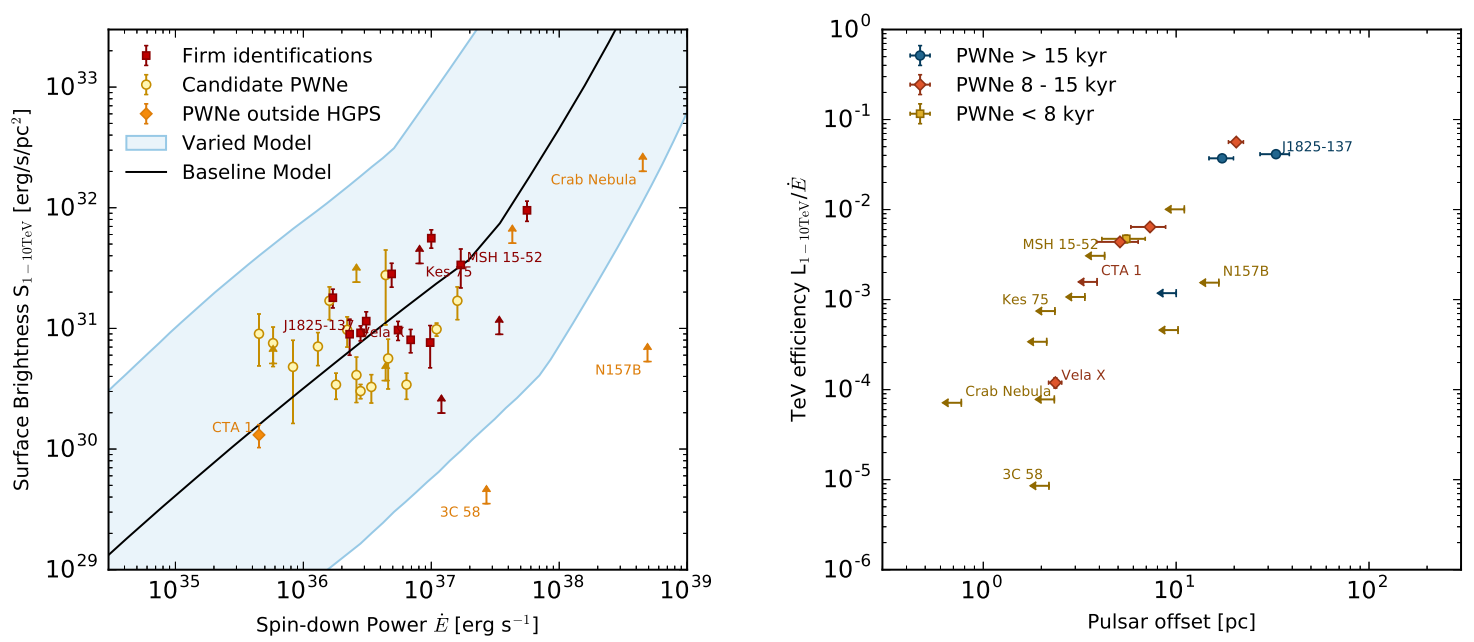

Figure 3: Left: Relation of TeV surface brightness and pulsar $\dot{E}$. Right: $\mathrm{TeV}$ efficiency as a function of pulsar offset, plotted for pulsars of different age groups. High-offset systems tend to be more TeV-efficient than low-offset systems. The figures are reproduced from H.E.S.S. Collaboration et al. 2017 [1].

The evolution trend of the photon index remains an open issue at present. Neither the data nor the model are particularly clear about it for the young to middle-aged PWNe we investigated.

\section{Detection biases}

Since both the H.E.S.S. Galactic Plane Survey and the ATNF pulsar database only cover a fraction of the Milky Way, depending on $\mathrm{TeV}$ and pulsar brightnesses, the study in H.E.S.S. Collaboration et al. 2017 [1] suffers from several selection biases discussed throughout the paper. For TeV-bright, high- $\dot{E}$, young pulsar systems $\left(>10^{36} \mathrm{erg} \mathrm{s}^{-1}\right.$ ) we achieve a relatively good coverage, whereas for systems beyond some tens of kiloyears of age we likely miss many sources. In the plots discussing flux-related quantities, this is partly compensated by the inclusion of flux limits, allowing for statements that consider the presence of non-detections. For extension- and position-related quantities, however, we can only rely on the detected cases. It would require a full population synthesis study to judge whether some of the correlations are genuine or include side effects of other correlations or selection biases. This usually needs many astrophysical assumptions and theoretical suppositions, which was beyond the scope of this experimental paper. 
One presumably very influential parameter ignored in this study is the density of matter and background light at the position of each pulsar. It is likely due to such circumstances that 3C 58, CTA 1 and Vela X, also presented at this conference [5] are so faint (see Fig. 1), and N 157B (in the Large Magellanic Cloud) is so bright. In the scope of a population synthesis study, one could use a specific Milky Way model to "calibrate" the calorimetric objects that $\mathrm{TeV}$ pulsar wind nebulae are assumed to be.

\section{Modelling}

On the modelling side, we are able to describe the trends and scatter of the $\mathrm{TeV}$ properties of the present PWN population with a relatively simple time-dependent modelling described in Appendix A of H.E.S.S. Collaboration et al. 2017 [1] and whose basic evolution is displayed in Fig. 4. Its 12 free parameters ( 7 of which were varied for the varied model) were well below the $4 \times 19$ observed parameters that the firmly identified PWNe provided. It is remarkable that the adaptive parameters needed to be varied in a fairly small range, compared to what one may fathom from the modelling literature, while still producing sufficient scatter in the predicted observables. Whether this indicates that the variations of the individual PWN parameters are indeed small, or whether this is an effect of the parameters being (anti-)correlated (see caveats discussion in A.7 of H.E.S.S. Collaboration et al. 2017 [1]), could not be clarified in this work. It might require a deeper physical model of the pulsars and possibly a multidimensional likelihood fit to correctly quantify all correlations and identify the true distributions of its parameters.
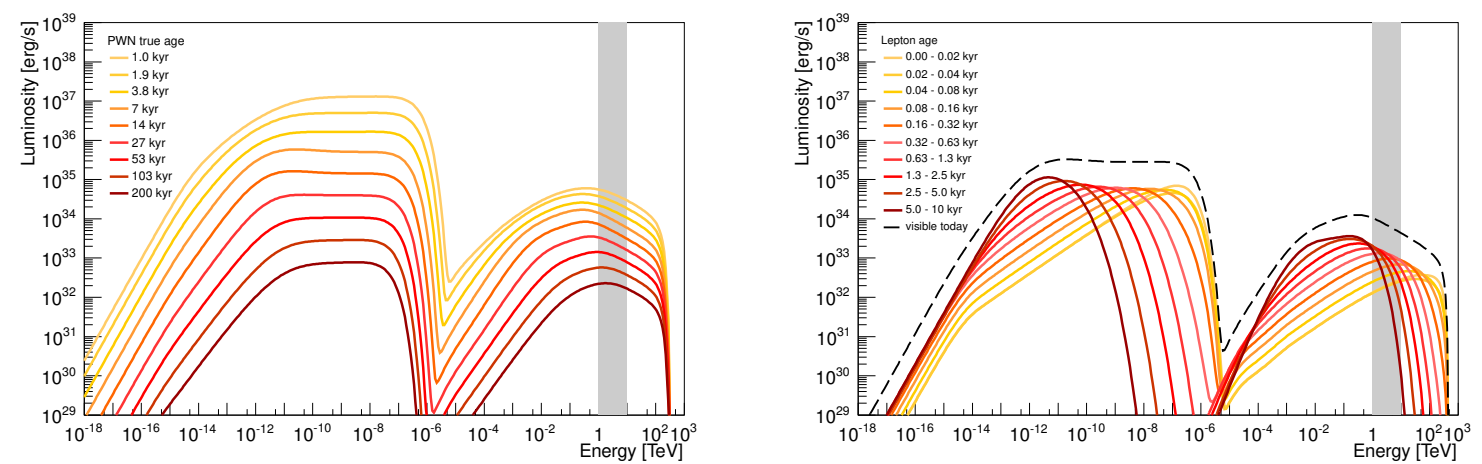

Figure 4: Modelled spectral energy distribution (SED) of a generic PWN according to the model given in [1], Appendix A. Left: Time evolution of the SED, ranging from 1 kyr to $200 \mathrm{kyr}$. Right: Decomposition of the SED of a middle-aged PWN (10 kyr; black dashed curve) into contributions by leptons from various injection epochs (coloured lines). The grey-shaded bands indicate the energy range of 1-10 TeV explored in this work. The figures are reproduced from H.E.S.S. Collaboration et al. 2017 [1].

\section{Outlook}

In the CTA era, many of the PWNe that will be detected in addition to the now assessed population will be middle-aged and old systems that are too faint or too extended to be detected with current instruments. Also, improvements in the radio and gamma-ray band coverage will 
enlarge the sample of pulsars detected in our Galaxy. To gain new insights from studying these systems, a solid and publicly available modelling code is needed that includes the difficult reverse shock interaction phase of a PWN in a reproducible way. This may help to understand the effect and influence of the amount of crushing and pulsar offset of the PWN, which is likely an influential factor of later PWN evolution.

On the analysis side, it would be beneficial to (i) improve the angular resolution and get to smaller scales of extension, (ii) find ways to reliably disentangle overlapping sources and their spectra, and (iii) aim for detecting objects larger than the IACT camera FOV. It is only if this is improved that larger datasets and more exposure can help us to uniddle sources that are closeby or occult each other in the densely populated arms of the Galaxy.

\section{Acknowledgments}

The support of the Namibian authorities and of the University of Namibia in facilitating the construction and operation of H.E.S.S. is gratefully acknowledged, as is the support by the German Ministry for Education and Research (BMBF), the Max Planck Society, the German Research Foundation (DFG), the French Ministry for Research, the CNRS-IN2P3 and the Astroparticle Interdisciplinary Programme of the CNRS, the U.K. Science and Technology Facilities Council (STFC), the IPNP of the Charles University, the Czech Science Foundation, the Polish Ministry of Science and Higher Education, the South African Department of Science and Technology and National Research Foundation, the University of Namibia, the Innsbruck University, the Austrian Science Fund (FWF), and the Austrian Federal Ministry for Science, Research and Economy, and by the University of Adelaide and the Australian Research Council. We appreciate the excellent work of the technical support staff in Berlin, Durham, Hamburg, Heidelberg, Palaiseau, Paris, Saclay, and in Namibia in the construction and operation of the equipment. This work benefitted from services provided by the H.E.S.S. Virtual Organisation, supported by the national resource providers of the EGI Federation.

\section{References}

[1] H.E.S.S. Collaboration et al., accepted for publication in $A \& A$, arXiv:1702.08280.

[2] H.E.S.S. Collaboration et al., Science 347406 (2015), arXiv:1501.06578.

[3] A.F. Aharonian et al. (H.E.S.S. Collaboration), A\&A 442 L25 (2005), arXiv:astro-ph/0510394.

[4] A.M.W. Mitchell et al., PoS ( ICRC2017) 707

[5] L. Tibaldo et al., PoS ( ICRC2017) 719 